\title{
Granular Controls on Periodicity of Stick-Slip Events: Kinematics and Force-Chains in an Experimental Fault
}

\author{
Nicholas W. Hayman, ${ }^{1}$ Lucie Ducloué,,${ }^{1,2}$ Kate L. Foco, ${ }^{3}$ and Karen E. Daniels ${ }^{3}$
}

\begin{abstract}
It is a long-standing question whether granular fault material such as gouge plays a major role in controlling fault dynamics such as seismicity and slip-periodicity. In both natural and experimental faults, granular materials resist shear and accommodate strain via interparticle friction, fracture toughness, fluid pressure, dilation, and interparticle rearrangements. Here, we isolate the effects of particle rearrangements on granular deformation through laboratory experiments. Within a sheared photoelastic granular aggregate at constant volume, we simultaneously visualize both particle-scale kinematics and interparticle forces, the latter taking the form of force-chains. We observe stickslip deformation and associated force drops during an overall strengthening of the shear zone. This strengthening regime provides insight into granular rheology and conditions of stick-slip periodicity, and may be qualitatively analogous to slip that accompanies longer term interseismic strengthening of natural faults. Of particular note is the observation that increasing the packing density increases the stiffness of the granular aggregate and decreases the damping (increases time-scales) during slip events. At relatively loose packing density, the slip displacements during the events follow an approximately power-law distribution, as opposed to an exponential distribution at higher packing density. The system exhibits switching between quasi-periodic and aperiodic slip behavior at all packing densities. Higher packing densities favor quasi-periodic behavior, with a longer time interval between aperiodic events than between quasi-periodic events. This difference in the time-scale of aperiodic stick-slip deformation is reflected in both the kinematics of interparticle slip and the forcechain dynamics: all major force-chain reorganizations are associated with aperiodic events. Our experiments conceptually link observations of natural fault dynamics with current models for granular stick-slip dynamics. We find that the stick-slip dynamics are consistent with a driven harmonic oscillator model with damping provided by an effective viscosity, and that shear-transformation-zone, jamming, and crackling noise theories provide insight into the effective stiffness and patterns of shear localization during deformation.
\end{abstract}

1 Institute for Geophysics, University of Texas, 10100 Burnet Rd., Bldg. 196, Austin, TX 78758-4445, USA. E-mail: hayman@utig.ig.utexas.edu

2 Département de Physique, École Normale Supérieure, 24 rue Lhomond, 75005 Paris, France.

3 Department of Physics, North Carolina State University, Raleigh, NC 27695, USA. E-mail: kdaniel@ncsu.edu

\section{Introduction}

Faults in the Earth's crust accommodate plate tectonic displacements, cause destructive earthquakes, and obey stick-slip mechanics similar to other processes such as landslides and glacial sliding. The spatial and temporal patterns of stick-slip behavior in natural faults are important both as a fundamental geophysical problem and for seismic hazard assessment. Some faults, such as segments of the San Andreas fault, appear to slip approximately periodically with self-similar, characteristic earthquakes (Schwartz and Coppersmith, 1984; Scharer et al., 2010). Additionally, the great subduction-zone earthquakes appear to have somewhat periodic recurrence intervals (Mogi, 1986; GoldFinger et al., 2003). Yet, even faults prone to characteristic earthquakes have variations in recurrence interval (MURRAY and SEgALL, 2002). Rather than loading a simple frictional faultplane to failure, the range of observed earthquake periodicity appears to derive from relationships between the evolving stresses that load faults and trigger earthquakes, and the mechanical response of structurally heterogeneous fault zones (STEIN et al., 1994; Chen et al., 2007; Ben-Zion, 2008). The mechanical response of fault zones may also play a disproportionately large role in governing fault-slip dynamics as shown by faults that either creep aseismically or with very subtle seismic signals (ROGERs and Dragert, 2003; Beroza and Ide, 2009). These events can be quite periodic (MiLLER et al., 2002; BRUDZINSKI and Allen, 2007) and are defined by longer durations and shorter slip distances than large seismic slip events (IDE et al., 2007; BRodSKY and Mori, 2007).

The different fault-slip dynamics are thought to have their origin in the frictional properties of faults (Scholz, 1998; Marone, 1998), modified by shear 
zone dilation and fluid pressure fluctuations (SEGALL and Rice, 1995; Segall et al., 2011). However, observations of natural fault materials (MOORE and RyMer, 2007; Schleicher et al., 2010) and experiments on fault gouge and fault-gouge analogs (SAFFER and Marone, 2003; Anthony and Marone, 2005; SMith and FAUlKNER, 2010) show that texture and composition of a granular mixture can exert a large influence on the strength and sliding behavior of a fault zone. A logical interpretation of such results is that the granular interactions strongly affect fault-slip dynamics, as also supported by discrete numerical models (Aharonov and Sparks, 1999; Morgan, 2004; Alonso-Marroquin et al., 2006; MaIr and HazZARD, 2007; ABE and MAIR, 2009). By granular interactions, we mean the elastic interactions between, and rearrangements of, particles during strain. Such interactions lead to highly heterogeneous spatial and temporal distributions of forces which are known to exist in laboratory granular materials (DANTU, 1954; Drescher et al., 1972; Liu et al., 1995; Miller et al., 1996). Recent advances in the physics of granular materials, while not specifically related to fault systems, have highlighted the importance of heterogeneous force distributions (van HeCKE, 2005), emphasized localization within shear transformation zones (FAlK and Langer, 2000; Daub and Carlson, 2010), and provided a framework for a general viscoelastic flow law for granular materials (FORTERRE and Pouliquen, 2008). Specific models and experiments stemming from the physics of granular materials have successfully reproduced power-law earthquake statistics such as the Gutenberg-Richter (G-R) relationship (CARLSON, 1991; DAHMEN et al., 1998; BRETZ et al., 2006; Yu, 2008; DANIELS and Hayman, 2008; Dahmen et al., 2009a, b) and periodic-aperiodic transitions (NASUNO et al., 1997, 1998; Daub and Carlson, 2009). Therefore, it is reasonable to suspect that the physics of granular materials offers insight into a broader range of natural fault behaviors.

In this paper, we present the results of a series of experiments wherein a quasi-two-dimensional aggregate of centimeter-scale photoelastic particles deforms within a constant-volume shear cell (see Fig. 1). Though the boundary conditions and overall friction evolution are quite different from more widely used fault-zone analogs, we access stick-slip
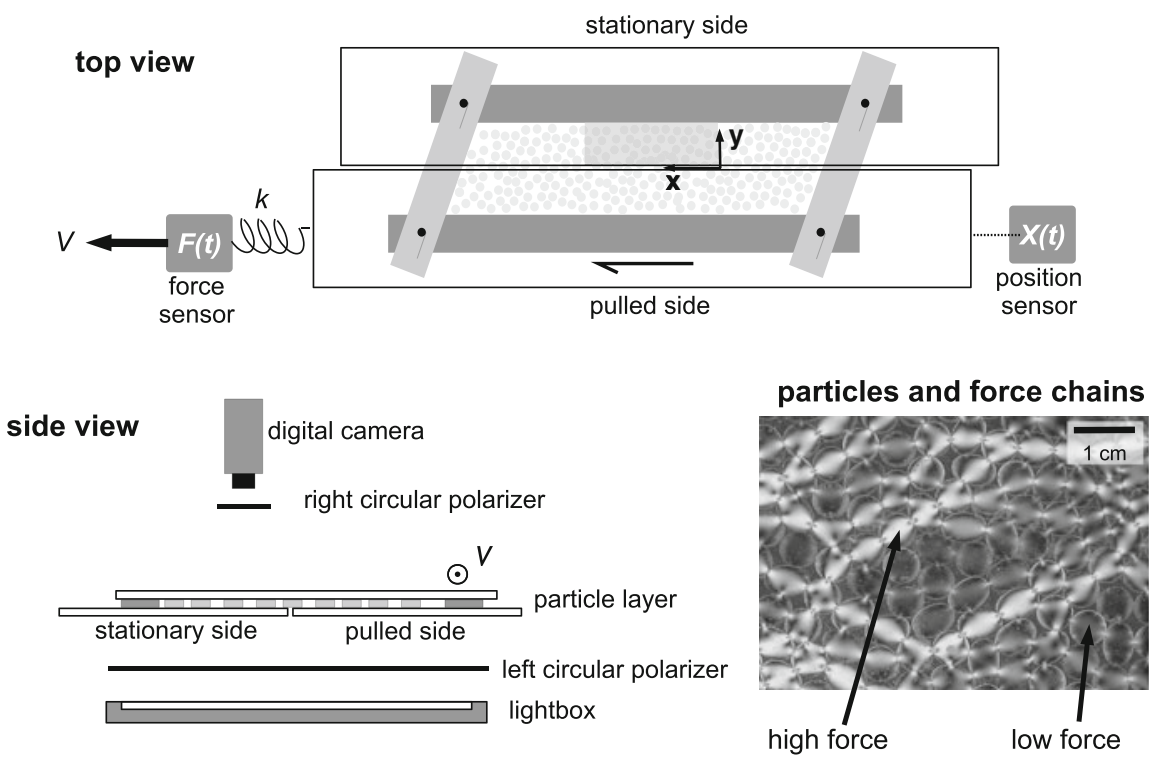

Figure 1

Schematic of laboratory fault apparatus (not to scale). Top view stationary side is fixed in place; pulled side is driven at constant velocity via a spring coupling (at left). Central region is filled with photoelastic disks, and the gray walls provide constant volume under shear. Shaded region corresponds to area shown in Fig. 5. Boundaries parallel to the shear (dark gray) are rough on the scale of the particles; end boundaries (light gray) are smooth. The pulled side and dark gray wall move as a single unit. Side view shows polariscope used to visualize force-chains.

Photograph of photoelastic particles with force-chains: brighter particles are those carrying more force 
regimes that have, to our knowledge, been largely overlooked but are likely relevant to natural faulting. Additionally, the strengthening that occurs in a constant volume shear cell could be compared to the interseismic, but still stick-slip, strengthening in natural faults. The use of photoelastic particles permits us to directly visualize the internal stresses. The stresses take the form of heterogeneously-distributed chains of strong force which, on average, align with the principal stress axes of the system. When one side of the aggregate is sheared with respect to the other across a split between the underlying plates, stresses build up until the force-chains lose stability and buckle (Howell et al., 1999; CATES et al., 1998; Tordesillas and Muthuswamy, 2009). This displacement and force drop can then be accompanied by either interparticle slip and force-chain buckling (local failure), bulk sliding of the aggregate (boundary failure), or a combination of the two. By varying the number of particles within the cell (mean packing density $\phi$ ), we adjust the degree of quasi-periodicity/ aperiodicity of the stick-slip events and explore the effects of packing density on kinematics and forcechain behavior. After presenting the details of our experiments and results, we interpret them in light of granular jamming, deformation, and rheology models, as well as their relationship to natural earthquake and faulting behaviors.

\section{Experiment}

The experimental apparatus consists of a springpulled slider block that deforms a photoelastic granular aggregate at a constant velocity and constant volume (see Fig. 1). The split in the center of the cell provides relative displacement of the two plates, and prevents the shear from localizing on the side boundaries instead of the center (FENISTEIN and van HeCKe, 2003). One plate is fixed and the other pulled by a stepper motor attached to a linear feed screw which moves at a constant velocity $V=0.30 \mathrm{~mm} / \mathrm{s}$. The plate is connected to the motor by a spring with stiffness $k=0.83 \mathrm{~N} / \mathrm{mm}$. The granular aggregate is a single horizontal layer of $60 \%$ circular (diameter $5.6 \mathrm{~mm}$ ) and $40 \%$ elliptical disks with approximately equal area, $\mathcal{O}\left(10^{4}\right)$ particles in total. The aggregate is confined to a monolayer by a solid plate. The particles interact with each other through non-cohesive, frictional interactions. As the slider block moves at constant velocity, it stretches the spring which couples it to the pulled plate and the force grows linearly with time (and motor displacement). When this pulling force exceeds the net frictional force along the imposed fault, the pulled plate begins to move forward until the frictional forces bring it to rest and the process begins again. These dynamics result in stick-slip behavior. Further details about the experimental apparatus are provided in DANIELS and HAYMAN (2008).

We measure the motion and the dynamics of the pulled plate using a resistive position sensor which monitors the location of the plate, $X(t)$, and a piezoelectric force sensor (coupled through the pulling spring) which measures the pulling force $F(t)$. Examples of each, at representative low and high packing density, are provided in Fig. 2. Note that not all internal stress is relieved during each event, resulting in an overall increase in $F$ over time (see Fig. 2a). This results in the position of the pulled plate lagging behind the slider block (see Fig. 3a). All analysis is performed on the system after it reaches the linear part of the force-displacement curve (see Fig. 3b), observed for $F>11 \mathrm{~N}$, independent of $\phi$. Every slip forward, $\Delta X$, is accompanied by a force drop, $\Delta F$, during duration, $\Delta T$.

In order to locate small events, we first remove electrical noise from $X(t)$ using a Wiener filtering technique (Papanikolaou et al., 2010). Using the product $\left(\frac{\mathrm{d} X}{\mathrm{~d} t}\right) \cdot\left(\frac{\mathrm{d} F}{\mathrm{~d} t}\right)$, we locate all peaks above a threshold magnitude and locate the start and end times of each event. Events smaller than this threshold are discarded from the analysis. For each event, we measure $\Delta X, \Delta T$, and the intervals preceding and succeeding each event ( $I_{\text {pre }}$ and $\left.I_{\text {post }}\right)$, shown, for example, in Fig. 2. Each measured event is system-spanning in the sense that it causes the full length of the imposed fault to rupture; local failure events which do not span the full length of the fault are not detected in this analysis.

The mean packing density $\phi$ is defined as the ratio of the area occupied by particles to the total available area. We explored the importance of $\phi$ on stick-slip rheology by varying the total number of particles in the system from 10,150 to 10,675 . Our experiments 
(a) $\phi=0.806$

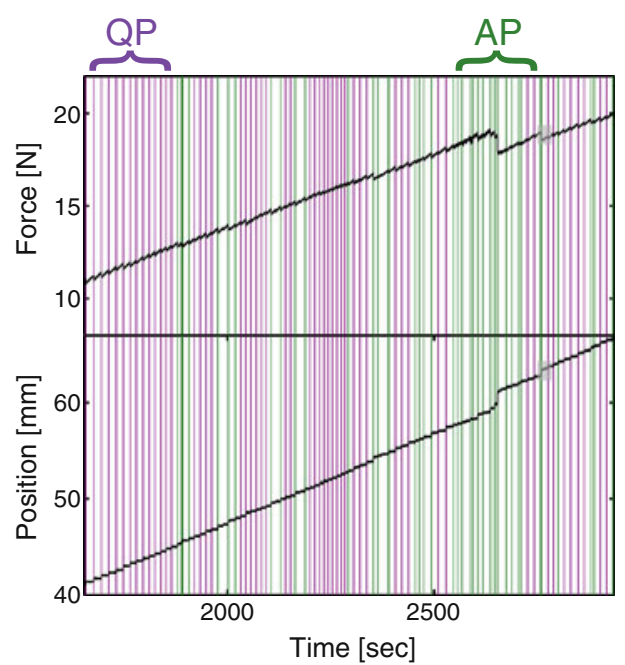

(b) $\phi=0.840$

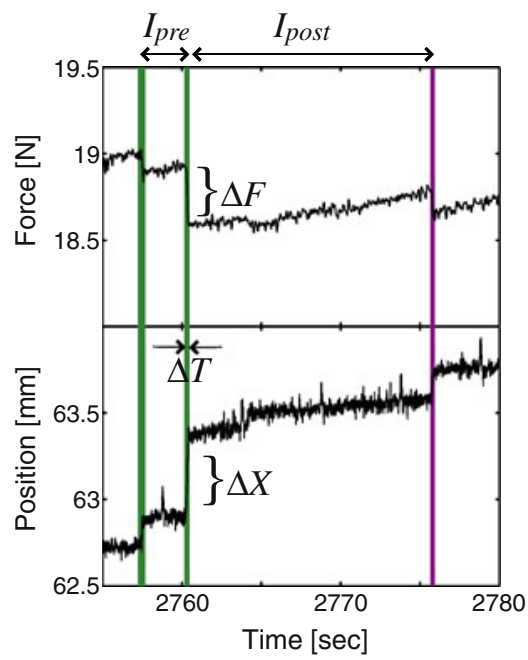

Figure 2

Sample $F(t)$ and $X(t)$ time series showing stick-slip events during constant-volume granular shear at two representative mean packing densities (a) $\phi=0.806$ and (b) $\phi=0.840$. Purple vertical lines mark quasi-periodic (QP) stick-slip events; green lines mark aperiodic (AP) stick-slip events. The center panel shows a magnified portion of the $\phi=0.806$ data, indicating the definitions of force drop $(\Delta F)$, the time-interval before $\left(I_{\text {pre }}\right)$ and after $\left(I_{\text {post }}\right)$ a stick-slip event with duration $(\Delta T)$ and total plate slip $(\Delta X)$

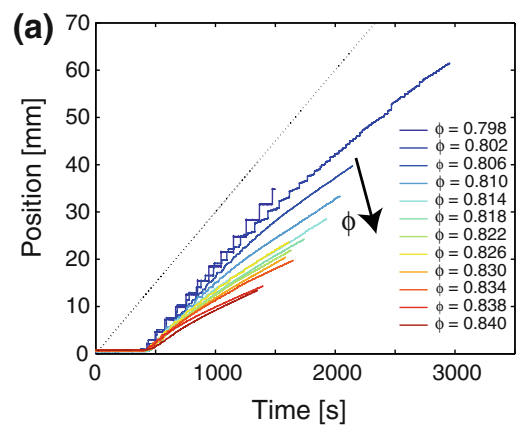

(c)

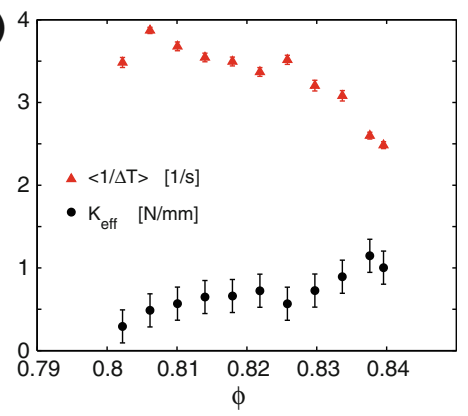

Figure 3

a Average pulled plate position $\langle X(t)\rangle$, averaged over all runs at the same $\phi$, specified by color. From low to high $\phi$, the number of runs in each average is $1,2,13,7,7,7,7,9,7,5,14,12$. Arrows show direction of increasing $\phi$. Dotted line is $X(t)=V t$ for pulling speed $V=0.3$ $\mathrm{mm} / \mathrm{s}$. b Parametric plot of average pulling force $\langle F(t)\rangle$ versus average pulled plate position $\langle X(t)\rangle$. Dotted line is $F_{0}=11 \mathrm{~N}$. c Effective elasticity of the aggregate $K_{\text {eff }}$, and damping $\propto\langle 1 / \Delta T\rangle$.

cover $0.798<\phi<0.840$. The lower end of this range generates behavior reminiscent of experiments tailored to large stress drops about a steady-state friction. The underlying reason for such relatively constant frictional behavior is that the system falls below the rigidity transition (random loose packing, $\left.\phi_{\mathrm{RLP}}\right)$, and is unable to sustain any permanent shear stress (ONODA and Liniger, 1990; Silbert, 2010). At the upper end of the range, the system is approaching random closed packing ( $\left.\phi_{\mathrm{RCP}}\right)$, above which it is not possible to insert another particle into the system without introducing crystallization, and no local rearrangements are possible, only global (SCOTT and Kilgour, 1969; Torquato et al., 2000). For frictional systems, the range of jammed (a.k.a. mechanically stable) states falls between $\phi_{\mathrm{RLP}}$ and $\phi_{\mathrm{RCP}}$ (BRISCOE et al., 2008).

As can be seen in Fig. 2, the system exhibits switching between quasi-periodic (QP) and aperiodic (AP) behavior, as was observed in model systems by 
DAhmen et al. (1998, 2009a, b) and Ben-Zion et al. (2010). We will refer to this behavior as periodicityswitching. We distinguish QP from AP modes by considering the time intervals preceding and succeeding each stick-slip event. If $I_{\text {pre }}$ and $I_{\text {post }}$ agree within $20 \%$ for a particular event, then that event is considered to be part of a QP series. Events for which $I_{\text {pre }}$ and $I_{\text {post }}$ differ by more than $20 \%$, as well as single QP events (those without adjacent QP events), are considered AP. The choice of $20 \%$ difference between $I_{\text {pre }}$ and $I_{\text {post }}$ is made in order to allow for a gradually-changing periodicity to be considered QP while not admitting AP events. Analysis of the same data with a threshold $\pm 5 \%$ does not affect the main trends observed. We calculate the periodic fraction $\alpha$ as the fraction of the total number of events which are designated QP. In the plots below, the variable I denotes the entire population of intervals between stick-slip events. When these intervals are plotted in reference to a particular event, then the variable name $I_{\text {pre }}$ or $I_{\text {post }}$ (see Fig. 2a) indicates whether the intervals are measured before or after the event, respectively.

In addition to the bulk dynamics, we isolate local failure dynamics such as interparticle slip and forcechain rearrangements with a pair of cameras mounted above the stationary side of the shear cell. A circular polarizing sheet is placed beneath the apparatus, and one of the two cameras is fitted with the opposite polarizing sheet in order to resolve the forces within each particle. The second camera simultaneously records the particle positions without a polarizing filter.

Particle kinematic measurements begin by locating the center of each particle in the non-polarized image with a Hough transform implemented using an open-source Matlab code. This method is able to detect both circular and elliptical particles. For each event detected from the bulk measurements, we compare particle positions $[x, y]$ immediately before and after the event, and determine the displacement $[\Delta x, \Delta y]$ of each using an open-source particle tracking code. Particle displacements range from 0.01 (our detection limit, based on pixel size) to $0.4 \mathrm{~mm}$. The collection of particle displacements defines a field with $\Delta x$ in the shear direction and $\Delta y$ in the spanwise direction. Note that variables relating to the bulk dynamics are denoted with capital letters and local, particle-scale kinematic measurements are denoted with lower case letters.

Additionally, for each event, we measure two kinematic descriptors of the displacement field: the mean local slip in the shear direction $\langle\Delta x\rangle$, and the mean gradient $\mathcal{G}$ of the displacement field. The former is calculated by taking the average over the ensemble of all particle displacements $\Delta x$. The mean gradient is calculated by first binning $\Delta x$ in the spanwise direction, to form $u(y)=\langle\Delta x\rangle(y)$, and then taking $\mathcal{G} \equiv\left\langle\left|\frac{\partial u}{\partial y}\right|\right\rangle$. The scalar $\mathcal{G}$ provides a useful measure of the extent to which the displacement field is solid-like (low $\mathcal{G}$ ) or exhibits internal deformation (high $\mathcal{G}$ ). The notation $\langle\cdot\rangle$ will used throughout the paper to indicate averages. A schematic definition of $\mathcal{G}$ is presented in Fig. 4; sample displacement vector fields are shown superposed on the initial frame in Fig. 5. The resolution of the images is $1,024 \times 1,280$ (about 30 pixels/particle), and each camera records the central region of the stationary side of the experiment. At the end of each run, each series of images (taken at $2 \mathrm{~Hz}$ ) is synchronized with the $X(t)$ and $F(t)$ signals described above. These local measurements are obtained for two representative packing densities, $\phi=0.806$ (5 runs) and $\phi=0.840$ (5 runs).

Force-chains build up in response to strain applied at the side and bottom boundaries, and are subject to three kinds of dynamics. First, because the shear stress on the system is increasing, the chains can become brighter (larger force) without moving. This is the dominant behavior during the interval between events. Second, for subsets of particles which slip together as a single unit (no contact failures or relative, interparticle displacements), the force-chains can slip with them. These are the boundary failures and appear in Fig. 5b as parallel white and black chains. Third, subsets of particles which have relative displacements with respect to each other are typically associated with force-chain buckling (TORDESILlas, 2007; Tordesillas and Muthuswamy, 2009). These are the local failures and appear in Fig. 5 as a branched network of black chains which are released, together with white chains which re-formed. We quantify the nature of the force-chain dynamics by examining the change in net photoelastic brightness 
(a) kinematic gradient

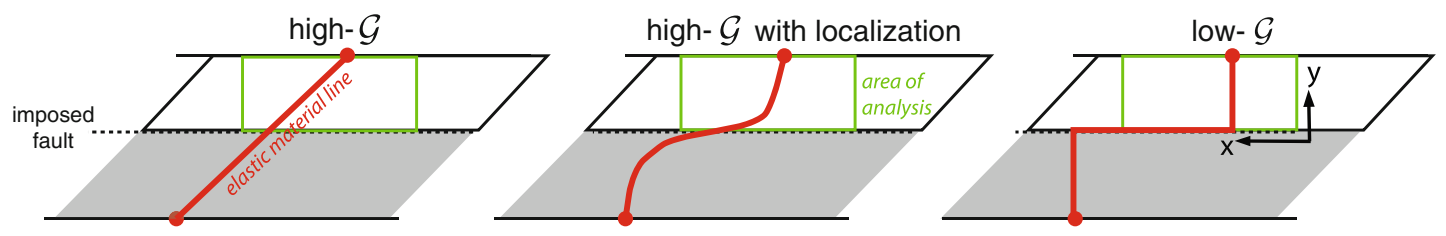

(b) image-differencing
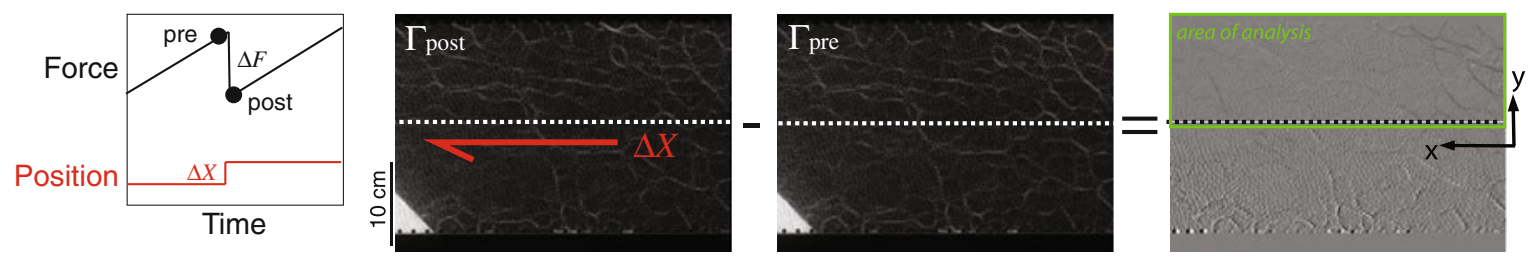

Figure 4

a Schematic definition of $\mathcal{G} \equiv\left\langle\left|\frac{\partial u}{\partial y}\right|\right\rangle$. For events where slip is accommodated by large interparticle displacements, $G$ is high. For events where uniform displacements of the entire aggregate accommodate slip, the mean kinematic gradient $G$ is low. b Sample event, showing force drop (schematic) and force-chain changes during a total plate slip $\Delta X$. Area of analysis corresponds to images in Fig. 5

averaged over particle-scale patches within the images $\Gamma_{\text {pre }}$ and $\Gamma_{\text {post }}$ preceding and following the event. The buckling/rearrangement events (local failure) produce a strong spatial signal, while boundary failure events where force-chains slip as a unit are weakly detected. These force-chain buckling patches are typically associated with patches of particles exhibiting local displacements in the non-shear direction. We quantify the degree of local failure relative to boundary failure via the failed fraction $\beta$, which is the fraction of the particle-scale patches which have $\Delta|\Gamma|$ above a threshold value.

In summary, for each event, we have four bulk characterizations (bulk event slip $\Delta X$, event duration $\Delta T$, and pre- and post-intervals $I_{\text {pre }}, I_{\text {post }}$ ) and three local, kinematic characterizations (average local slip $\langle\Delta x\rangle$, mean gradient $\mathcal{G}$, failed fraction $\beta$. In addition, each event is assigned a status as being part of a QP or AP series of events. In Sect. 3, we will consider how these bulk and local characterizations depend on packing density $\phi$ and the periodicity.

\section{Results}

\subsection{Rheology}

Several observations drawn from these dynamics provide insight into granular rheology, with implications for natural faulting. As shown in Fig. 3, the granular aggregate becomes stiffer over a shorter time for closely packed systems than for loosely packed systems. This strengthening arises because each stick-slip event only releases a fraction of the accumulated stress. We note that exploring changes in rheology does not come at the expense of a study of the stick-slip behavior: slip events exhibit stationary statistical distributions for $\Delta F$ and $\Delta X$ during the strengthening process (DANIELS and HAYMAN, 2008).

We quantify the effect of $\phi$ on the granular rheology by averaging together runs at the same $\phi$ to measure average behaviors $\langle X(t)\rangle$ and $\langle F(t)\rangle$. For $\phi \lesssim 0.8$, the granular aggregate repeatedly builds up a small amount of internal stress (as force-chains) and releases it in a periodic fashion without ever reaching a globally jammed configuration (O'Hern et al., 2003; Wyart, 2005). As such, $X(t)=V t$ on average, as seen by comparison with the slope of the dotted line in Fig. 3a. For $\phi \gtrsim 0.8$, the position of the plate falls behind this constant pulling rate, so that the slope of $X(t)$ is less than $V=0.3 \mathrm{~mm} / \mathrm{s}$. This effect increases as $\phi$ approaches $\phi_{\mathrm{RCP}}$.

In Fig. 3b, we plot $\langle F(t)\rangle$ as a function of $\langle X(t)\rangle$, parameterized by time. A linear regime, with stress proportional to strain, is found for all $\phi$ above a threshold force $F_{0}=11 \mathrm{~N}$, shown by the horizontal dotted line. In calculating statistics describing the 
(a) low $\phi(0.806)$, low $\mathcal{G}(0.048), \beta=3.9 \times 10^{-4}$
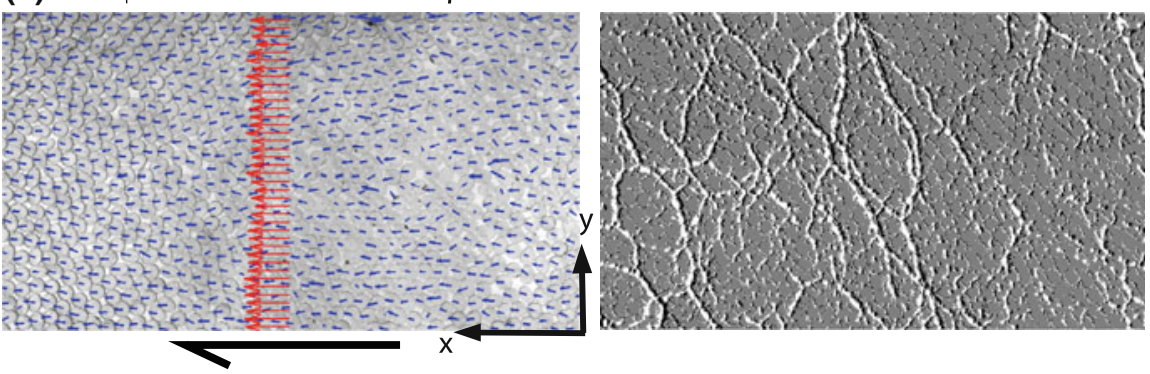

(b) low $\phi(0.806)$, high $\mathcal{G}(0.107) ; \beta=0.019$
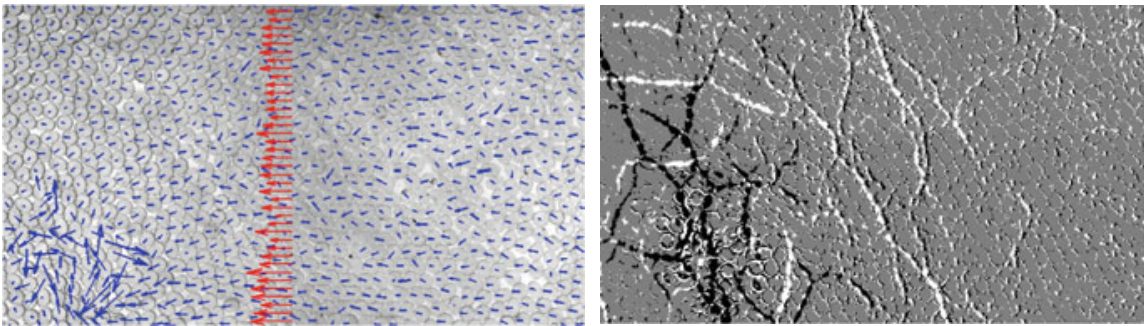

(c) high $\phi(0.84)$, low $\mathcal{G}(0.059), \beta=2 \times 10^{-4}$
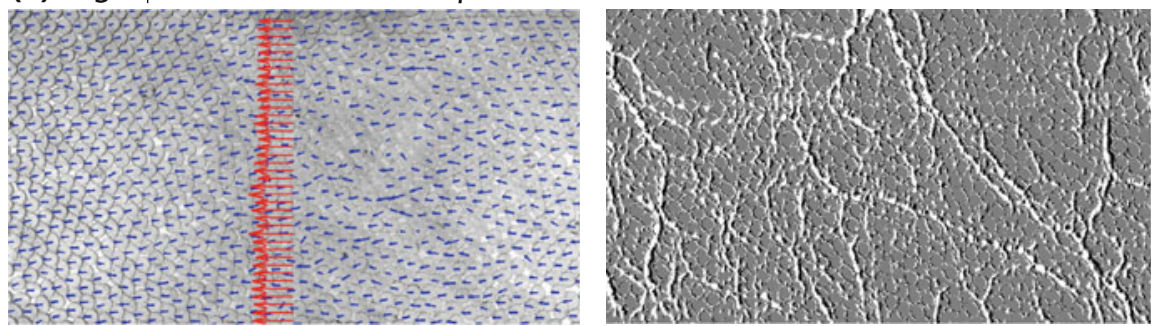

(d) high $\phi$ (0.84), high $\mathcal{G}(0.088), \beta=0$

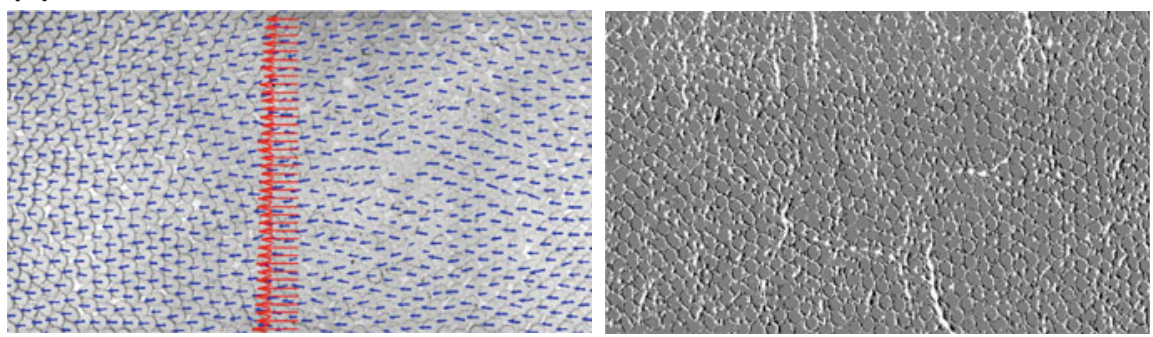

Figure 5

Images showing particle kinematics and force-chain dynamics for representative stick-slip events. In particle-kinematic images (left column), the blue arrows on each particle are the displacement field $[\Delta x, \Delta y]$ and the red line of arrows at the center are $u(y)$ used to calculate $\mathcal{G} \equiv\left\langle\left\langle\frac{\partial u}{\partial y} \mid\right\rangle\right.$. The force-chain images (right columns) are difference images $\Gamma_{\text {post }}-\Gamma_{\text {pre }}$ (see Fig. 4 ) wherein brighter chains strengthened, darker chains weakened, and bright chains immediately adjacent to darker chains mark a boundary failure in which the aggregate displaces while contacts remain intact. a Low $\mathcal{G}$ stick-slip event at low $\phi$ exhibiting predominantly boundary failure. b High $\mathcal{G}$ event at low $\phi$ exhibiting local failure with a network of force-chains failing in the vicinity of disordered particle rearrangements. c Low $\mathcal{G}$ stick-slip event at high $\phi$ exhibiting predominantly boundary failure. $\mathbf{d}$ High $\mathcal{G}$ stick-slip event at high $\phi$ exhibiting no large force-chain buckling or rearrangements

stick-slip events, we will only use data from this regime. We measure the effective stiffness $K_{\text {eff }}$ of the aggregate by making a linear fit to $F(X)$ above $F_{0}$. Figure $3 \mathrm{c}$ shows that $K_{\text {eff }}$ increases with $\phi$, as expected from numerical simulations by AHARONOV and Sparks (1999). In Sect. 4, we will discuss the implications of this change in rheology as a function of $\phi$. 


\subsection{Bulk event statistics}

Though stick-slip events maintain stationary distributions during strengthening during shear, changing the stiffness $K_{\text {eff }}$ of the system by increasing $\phi$ leads to corresponding changes in the statistics of the stick-slip events. For low $\phi$ (near unjamming), we observe power-law distributions of displacements and irregular event spacing. For high $\phi$, we observe more uniform events with more regular event spacing. In Fig. 6, we quantify this effect by examining the probability distributions of the three key event statistics, $\Delta X, \Delta T$, and $I . \Delta X$ shows power-law-like tails (similar to $\mathrm{G}-\mathrm{R}$ behavior) at lower $\phi$. The exponent of this distribution increases with $\phi$, eventually becoming more exponential-like (not shown) at the highest $\phi$. Similar changes in the size distribution were also observed for constant pressure vs. constant volume boundary conditions in the same apparatus (Daniels and Hayman, 2008). Meanwhile, $\Delta T$ of the events increases with $\phi$, while also becoming more broadly-distributed (also see Fig. $3 \mathrm{c}$ for the mean behavior). This indicates that an effective damping is decreasing with $\phi$ : once set in motion, the event is slower to stop. This effect will be discussed in more detail in Sect. 4. I does not vary significantly with $\phi$, suggesting that a characteristic timescale is present.
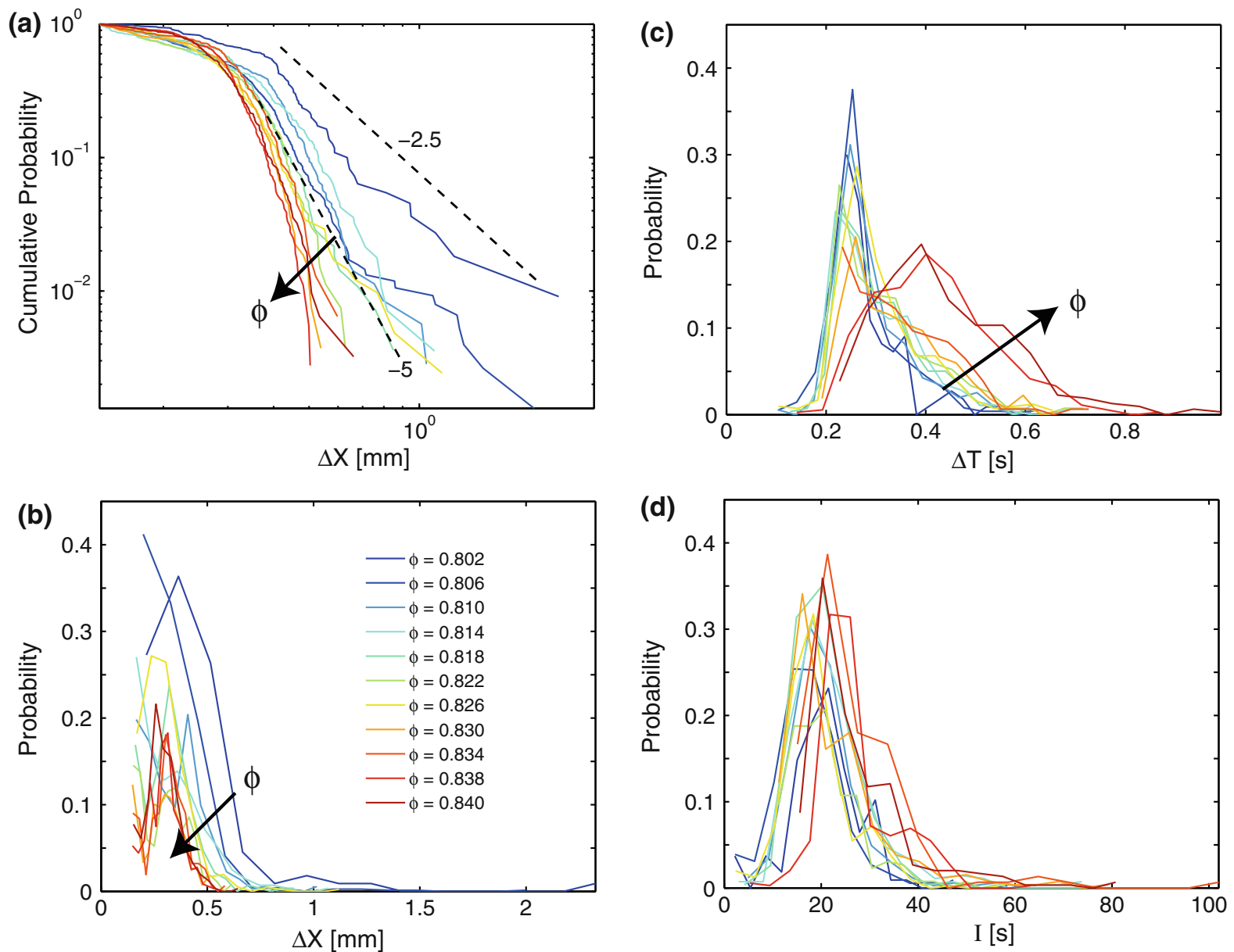

Figure 6

a Cumulative probability distribution of global slips $\Delta X$, with two power-laws $(-2.5$ and -5$)$ shown for comparison. b-d Probability density for global slips $\Delta X$, duration $\Delta T$ and interval $I$. All use the same color map from $\phi_{\mathrm{RLP}}$ (blue) to $\phi_{\mathrm{RCP}}(\mathrm{red})$, with trend from low to high $\phi$ indicated by the arrows 
We separate each of the probability distributions of Fig. 6 by sorting the events into two sub-populations, one containing all QP events and the other all AP events. As shown in Fig. 7a, we find that the fraction $\alpha$ of QP events increases with $\phi$. Independent of $\phi$, we find that the QP and AP modes exhibit indistinguishable distributions $\Delta X$ and $\Delta T$. However, $I$ is sensitive to whether the event is QP or AP. As $\phi$ increases, the AP events exhibit increasingly longer intervals, while the QP events retain the characteristic timescale shown in Fig. 6d.

\subsection{Particle kinematics and force-chain dynamics}

For two representative packing densities, $\phi$ $=0.806$ and 0.840 , we examine the particle and force-chain dynamics which are responsible for the bulk dynamics and the event statistics $\Delta X, \Delta T, I$, and
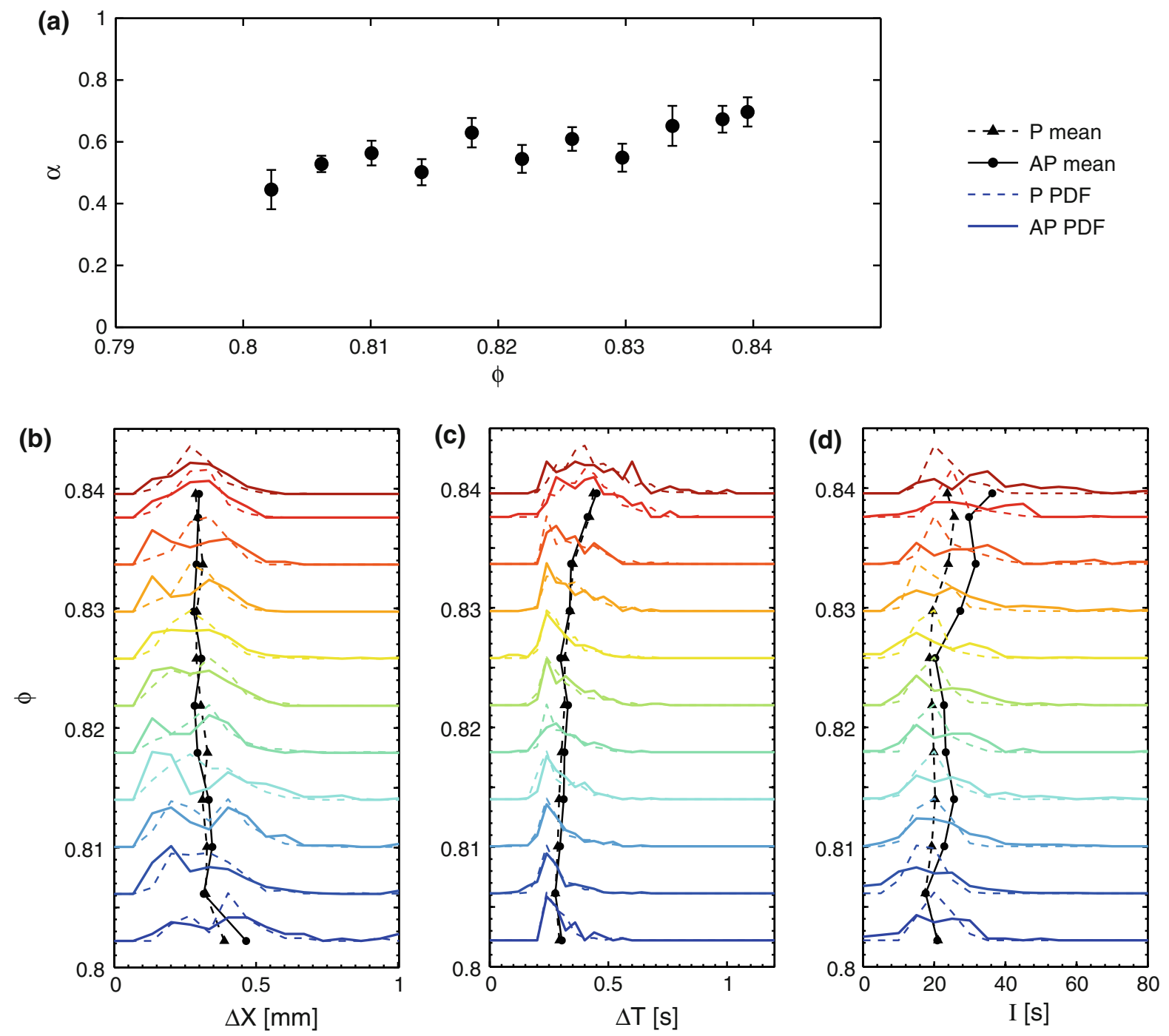

Figure 7

a Fraction $\alpha$ of events which are part of a QP series. Bars are standard error. b-d Probability density functions for $\Delta X, \Delta T$, and $I$, separated by both $\phi$ (color vertical displacement) and status as part of a quasi-periodic (QP dashed) or aperiodic (AP solid) series. The mean of the QP (filled triangle) and AP (filled circle) distributions is plotted as a function of $\phi$ 
QP/AP periodicity. In all cases, the forces are heterogeneously distributed, with the chains developing more quickly at large $\phi$, as indicated in the bulk dynamics in Fig. 3b. The local properties, observable in Fig. 5, are quantified via the average local slip $\langle\Delta x\rangle$, mean gradient $\mathcal{G}$, and failed fraction $\beta$. To understand the relationship between these variables in terms of $\phi$ and periodicity, we bin the data into six equally populated subsets and look at comparisons of average values (e.g. plot $\Delta X$ vs. $\mathcal{G}$ ).
Figures 8, 9, 10 present the most instructive of these comparisons. Figure 8 shows the pairwise comparisons for bulk $\left(\Delta X, I_{\text {pre }}\right)$ versus local $(\Delta x, \mathcal{G})$ properties, in order to investigate the effect of $\phi$ on local kinematics. Figures 9 and 10 then consider high and low $\phi$ in separate plots in order to isolate the periodicity related to the local kinematics.

Since all of the particles in the system remain within the constant volume region, we expect $\langle\Delta x\rangle \equiv$ $\Delta X$ on average. However, both measurements
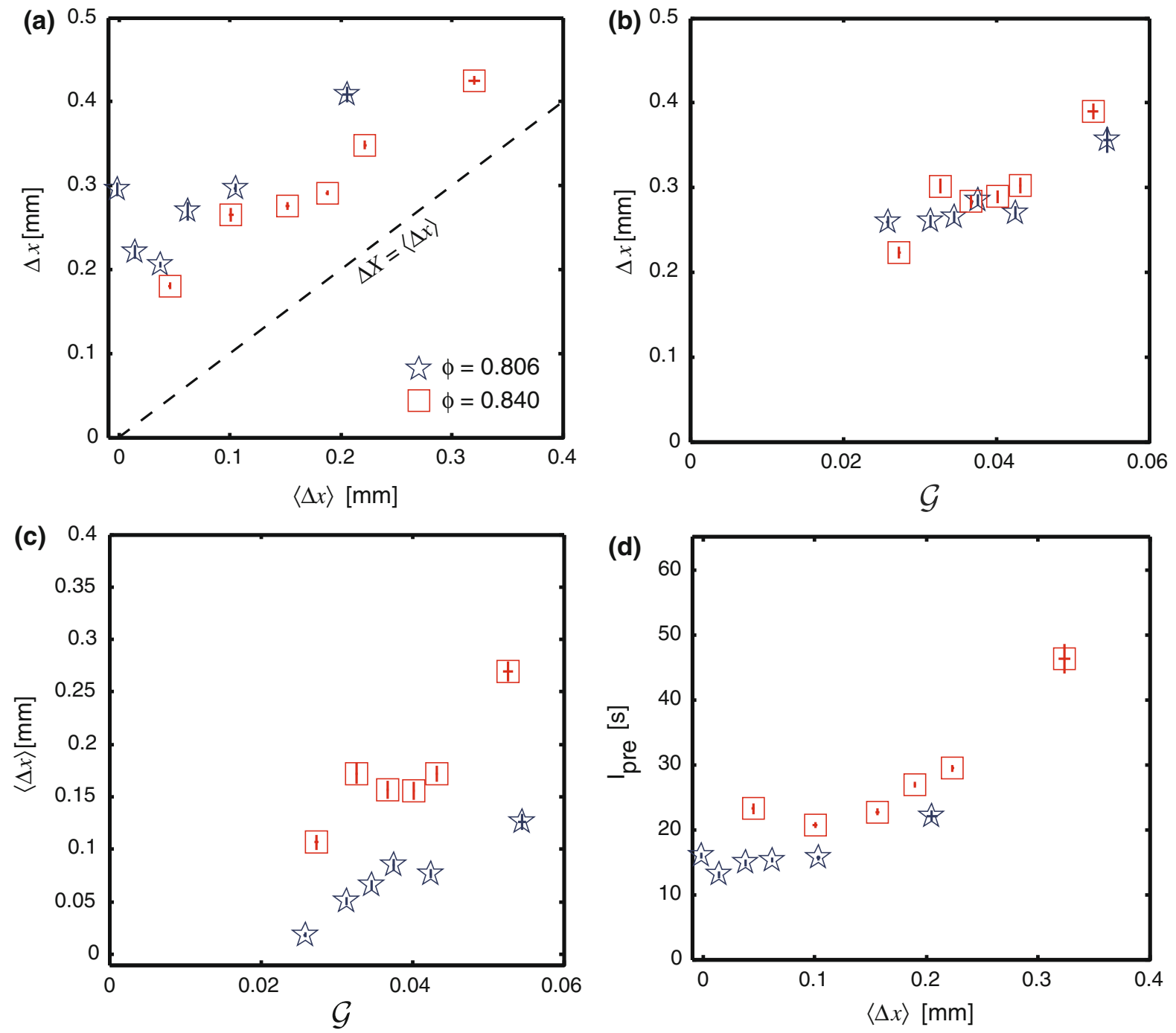

Figure 8

Dependence on $\phi$ of the relationships between: a global slip and mean local slip, b global slip and mean gradient, $\mathbf{c}$ mean local slip and mean gradient, and $\mathbf{d}$ preceding interval and mean local slip. On a, the dotted line is $\Delta X=\langle\Delta x\rangle$. Points at each $\phi$ are approximately equally populated when sorted by the abscissa. Bars show the standard error. For $\phi=0.806$ (asterisk), there are 292 total events and 0.840 (open square) has 129 total events 
exclude the smallest slips in practice; as shown in Fig. 8a, this equivalency is only approximately true even when averaged over a whole run. We observe that the relationship between $\Delta X$ and $\langle\Delta x\rangle$ is approximately linear, with a slope of 1 .

In the presence of shear-banding near the imposed fault, there is a gradient in the displacement field. As shown in Fig. $8 \mathrm{~b}$ and c, $\mathcal{G}$ exhibits a monotonic dependence on the bulk slip $\Delta X$ which is insensitive to $\phi$, while the local $\langle\Delta x\rangle$ is monotonic with a $\phi$ dependence. Thus, larger local slip events also exhibit larger $\mathcal{G}$, as would be expected for low-slip lateral boundaries. However, in systems with larger
$\phi,\langle\Delta x\rangle$ is also larger for a given $\mathcal{G}$. We interpret this somewhat counterintuitive result as arising from different types of internal deformation present at different packing density: low $\phi$ events are more likely to involve patches of disordered rearrangements (high $\beta$ ) than high $\phi$ events. This effect will be discussed in more detail in Sect. 5.

As already shown in Figs. 6d and 7d, $I$ grows somewhat with $\phi$. In Fig. 8d, we additionally observe that increased $I_{\text {pre }}$ is associated with increased $\langle\Delta x\rangle$. This may also be an effect of the increased degree of rearrangement present at low $\phi$ (see Fig. 5; a more detailed discussion of this trend follows in Sect. 3.5),
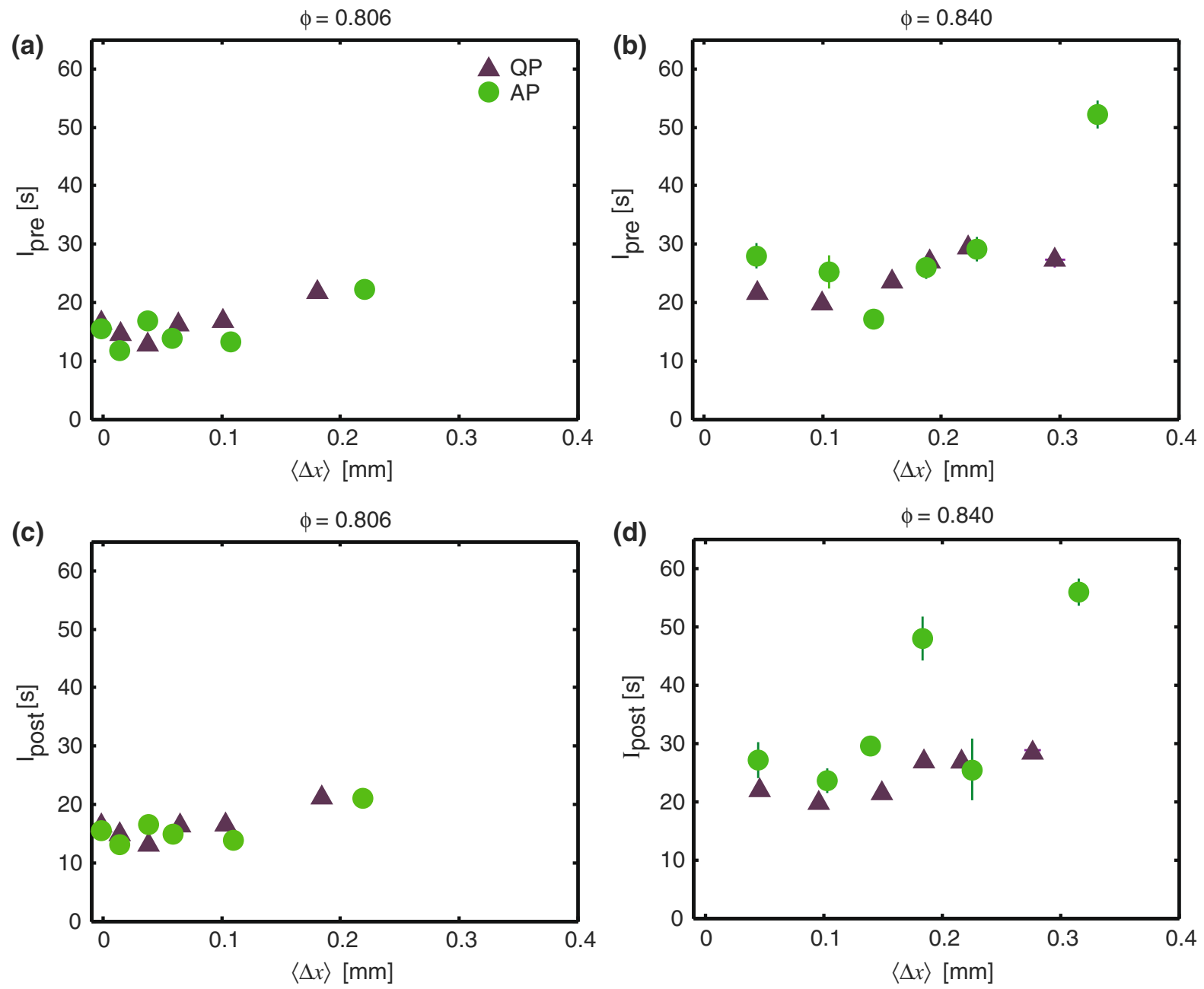

Figure 9

Relationship between mean local slip, $\langle\Delta x\rangle$, and (a, b) pre- and (c, d) post-interval, at low (a, c) and high $(\mathbf{b}, \mathbf{d})$ packing densities, $\phi$, with QP (filled triangle) and AP (filled circle) events plotted separately. Bars show the standard error. Points at each ( $\phi$, QP/AP) are approximately equally-populated when sorted by the abscissa 

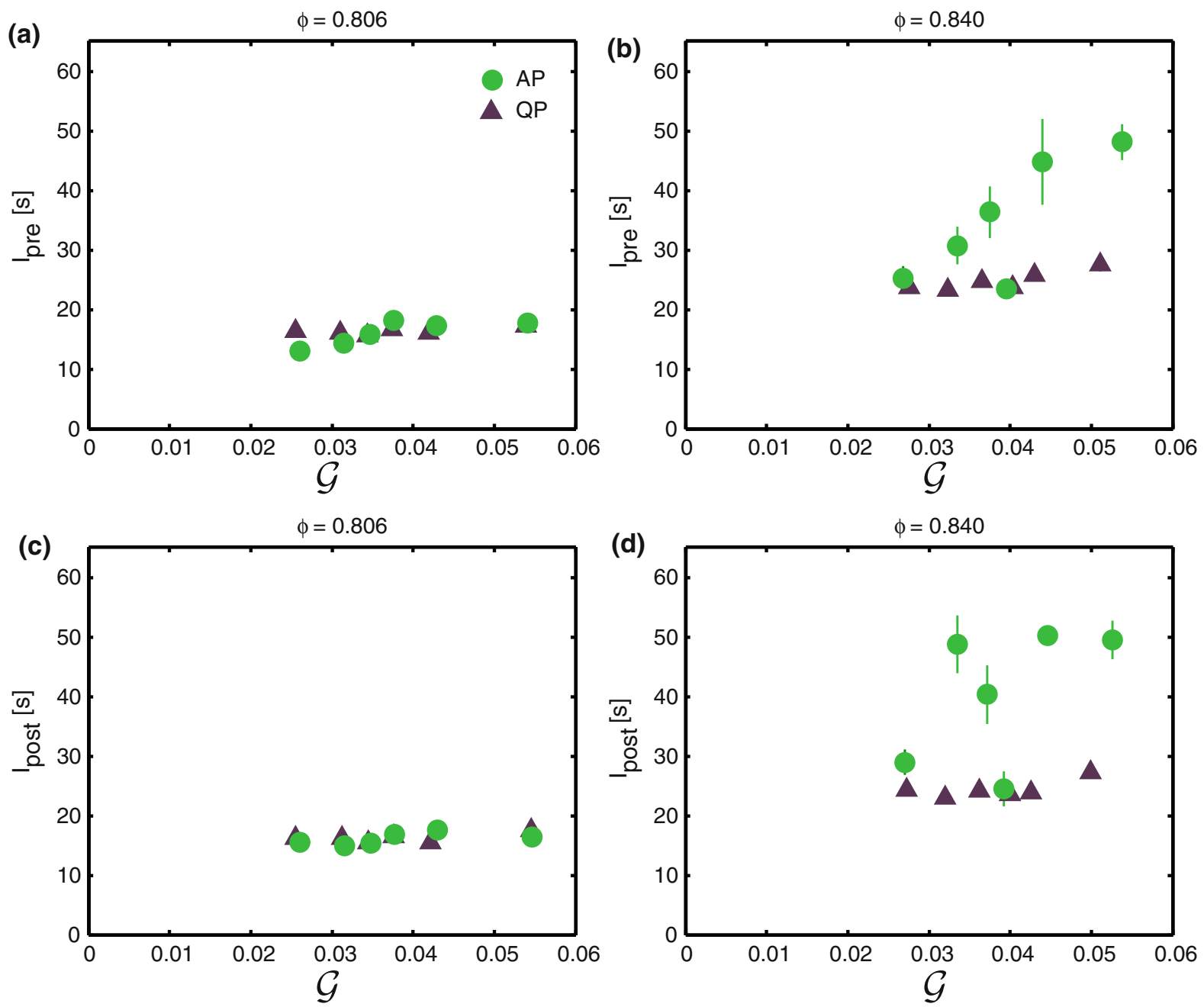

Figure 10

Relationship between gradient, $\mathcal{G}$, and $(\mathbf{a}, \mathbf{b})$ pre- and $(\mathbf{c}, \mathbf{d})$ post-interval, at low $(\mathbf{a}, \mathbf{c})$ and high $(\mathbf{b}, \mathbf{d})$ packing densities, $\phi$. Symbols and binning are the same as in Fig. 9

requiring a longer time between events to reform the strong force-chains which were broken. $I_{\text {post }}$ displays a similar trend (not shown), perhaps due to the quasiperiodicity of the system which makes $I_{\text {pre }} \approx I_{\text {post }}$.

\subsection{Periodicity}

A striking distinction between the events at low and high $\phi$ is their transition from more aperiodic (AP) to more quasi-periodic (QP) behavior. As shown in Fig. 9b and d, at high $\phi$ the dependence of $I_{\text {pre }}$ on $\langle\Delta x\rangle$ is different for AP and QP events. We observe that $I_{\text {pre }}$ and $I_{\text {post }}$ are typically longer for AP events with larger local displacement, but only for high $\phi$ (see Fig. 9a and c for low $\phi$ behavior). The outliers in the high $\phi$ data may be due to either failure events which occurred outside the area of observation or unusual large/small events. The relationship between periodicity and local particle kinematics does not on its own resolve the underlying dynamics of periodicity, but does provide insight into distinctions between AP and QP events. We will discus the influence of force-chains dynamics on AP and QP slip behaviors in the following section.

Another way of probing the relationship between periodicity and kinematics is to consider the mean 
kinematic gradient, $\mathcal{G}$. By definition, this measurement would be larger for events with larger $\Delta X$ as long as there was little slip at the lateral sidewalls. As shown in Fig. 10, similar trends are present for $\mathcal{G}$ as were observed for $\langle\Delta x\rangle$. At lower $\phi$, similar values of $\langle\Delta x\rangle$ and $I$ are observed, independent of $\mathcal{G}$ and periodicity. However, higher $\phi$ experiments have a population of AP, high $\mathcal{G}$ events with longer intervals than the corresponding QP events. The interval for these higher $\mathcal{G}$ events increases with $\mathcal{G}$. Note that this trend can also be observed in Fig. 7 d.

\subsection{Force-chain failure}

We have seen that local failures, as measured by tracking particle kinematics, play a key role in distinguishing AP and QP event statistics, and that these effects are additionally $\phi$-dependent. The relative displacements of adjacent particles are readily observed by the failure of force-chains, as shown in Fig. 10b. We more directly quantify these behaviors with $\beta$, which measures what proportion of an image participated in a major force-chain change or failure. As $\beta \rightarrow 0$, all force-chains in the imaged region retain their initial configuration, and failure is accommodated by boundary slip. As $\beta$ increases, a larger portion of the region participates in a local failure event, and failure is accommodated by both boundary and local failure. As shown in Fig. 11, at both high and low $\phi$, the AP events can involve a

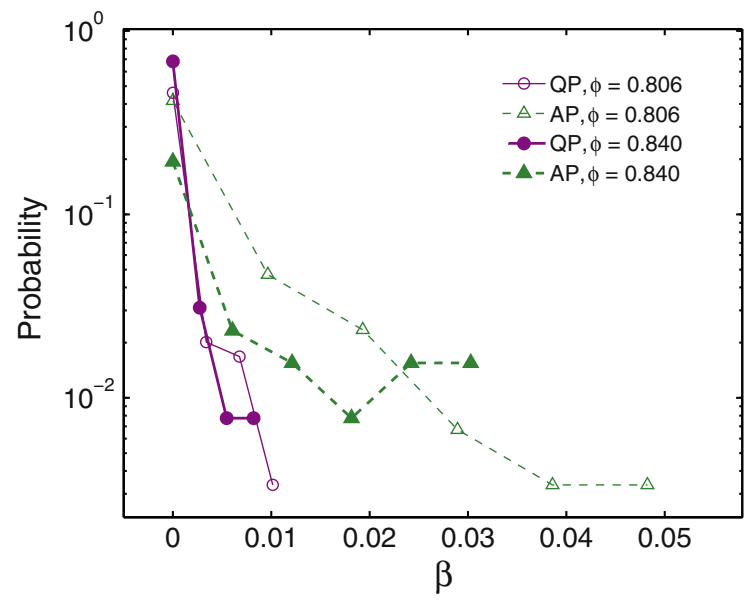

Figure 11

Histogram of failed fraction $\beta$, separated by packing density and periodicity significantly larger failed fraction of particles than the $\mathrm{QP}$ events. In fact, no QP events are observed to display significant local failure (large $\beta$ ), and all major force-chain reorganizations are associated with aperiodic events. In each case, there is a steep decline in the probability with $\beta$ : for no events do we see $\beta$ larger than $5 \%$ of the particles.

\section{Discussion}

Granular rearrangements, or configurational effects, are common to all particulate systems, causing them to resist shear even in the absence of other frictional forces (O'Hern et al., 2002). Natural faults may be sensitive to this granular, configurational effect. Most fault gouge is a mixture of materials with variable frictional properties and strength, suggesting that there are additional controls on fault strength and seismicity other than the properties of individual mineral constituents (SAFFer and MARONE, 2003). In turn, faults exhibit a range of seismic and aseismic behaviors, with varying periodicity and aperiodicity (IDE et al., 2007; Scharer et al., 2010). The traditional explanation for heterogeneous fault behavior is that velocity-weakening and -strengthening materials interact in the upper crust, and viscous and frictional materials interact in the deeper crust (KoHLSTEDT et al., 1995). In turn, fluids affect the mineralogy and microstructure of faults (WINTSCH et al., 1995; SCHLEICHER et al., 2010), and fluid pressure changes dynamically along with frictional properties (SEGALL and Rice, 1995; Segall et al., 2011). Yet, the properties (e.g., roughness, sizes, shapes) of granular fault materials can greatly affect fault-slip dynamics in isolation of other effects (ANTHONY and MARONE, 2005; MaIr and HaZzard, 2007; ABE and MaIr, 2009); this suggests the importance of isolating the effects of granular rearrangements themselves.

Our experiments are tailored to investigate the relationship between granular rearrangements and frictional dynamics. We report on a constant volume deformation regime which differs from other stickslip experiments that attempt to define a steady-state friction about which rate-and-state frictional behavior is established. Accessing this relatively unexplored strengthening regime has allowed us to better 
understand granular rheology. Furthermore, it may have a qualitative analog in the interseismic period of large earthquake producing faults, during which a wide range of stick-slip behavior is observed. However, our experiments are relatively restricted in the length and time scales of stick-slip deformation, and therefore cannot yet explore the range of durations and slip lengths that define different creep and seismic slip behaviors. In light of the limitations, it is noteworthy that we are able to determine statistically robust differences in duration (within a narrow range) of stick-slip events by increasing the packing density as shown in Fig. 10c. The change in duration is part of an overall effect of increasing packing density that includes increasing the elastic stiffness and decreasing the proportion of aperiodic events. Periodicityswitching is especially interesting because existing friction laws do not, on their own, describe controls on periodic and aperiodic behavior. Therefore, we extend this discussion to include some general theory surrounding granular deformation that bears on both periodicity and granular rheology of natural fault zones.

In considering the deformation of granular materials it is important to note that stick-slip dynamics fall at the boundary between two types of behaviors: solid-like and liquid-like. One difficulty in understanding such behaviors lies in creating a model that captures both the end of the 'stick' state when the system starts to flow, and the end of the 'slip' state as the system comes to rest. Three theoretical frameworks-shear transformation zones (STZ), jamming, crackling/avalanching — are making progress at addressing these issues.

\subsection{Shear transformation zones (STZ)}

The central idea of STZ theory is that particular regions of a disordered material are more susceptible to particle rearrangements (plastic failure, non-affine deformation) by virtue of being more disordered (FAlk and LANGer, 2000; Daub and Carlson, 2010). As a system is sheared, each new configuration is reached via a local change in particle configuration at one of the STZs. There is some energetic cost to performing these rearrangements, both from frictional interactions and from the elastic energy for particles to deform each other. During each waiting interval $I$, the system is subject to elastic (affine) deformation due to the shear. This shear causes the system to explore nearby metastable states in much the same way that thermal energy can excite molecular-scale systems from one state to another. For each stick-slip event (during $\Delta T$ ), the system undergoes plastic (non-affine) rearrangements and ends up in a new configuration which persists for the next $I$ interval. STZ theory has been successful in describing stick-slip dynamics (DAUB and CARLSON, 2009) and fault rupture (DAUB et al., 2010).

\subsection{Jamming}

The jamming framework describes how particulate materials arrive at mechanical stability as a function of $\phi$, confining pressure, and coordination number (van Hecke, 2010; Liu and Nagel, 2010). Near the jamming transition at $\phi_{c}$ (corresponds to $\phi_{\text {RLP }}$ for frictional systems), numerical simulations observe an excess of low frequency modes in the vibrational spectrum as compared to ordinary elastic solids. These soft modes provide a low-energy means to deform the aggregate, and provide a mechanism for transitions from each solid-like state to the next without the system ever becoming liquid-like. The length scale of these soft modes diverges as $\phi \rightarrow \phi_{c}$ from above. In contrast, for $\phi \gg \phi_{c}$, low-energy modes are no longer as prevalent.

\subsection{Crackling/avalanching}

Many physically distinct systems, ranging from disease epidemics to power grid outages, exhibit failure events which span a broad (power-law) range of sizes. Such behavior has come to be known as crackling, and can arise when a disordered system of interacting elements is driven to failure by external forcing (Sethna et al., 2001). If the disorder is a stronger effect than the interactions, then the system fails by many small individual failures. If the interactions are stronger than the disorder, then the system fails all at once. Crackling happens when the disorder and the degree of interaction are both important effects. 
Different aspects of these three theories apply to our experimental results. Each of the events in our experiments results from a rupture which propagates to span the full length of the fault, but can take two different forms. The boundary failure events occur without significant plastic deformation (observed within the region for which images are collected). The local failure events (see Fig. 10b) occur when a force-chain collapses (exceeds the Coulomb criterion locally) and the nearby particles rearrange. The frequency of local failures depends on packing density $\phi$, and the dynamics exhibit several STZ-like features. At lower $\phi$, as the system is sheared it is commonly able to find a new energy-minimum (metastable state) which is accessible via a STZ-like rearrangement. At larger $\phi$, it is more difficult to find nearby valid configurations, subject to the mechanical constraints of the system: this corresponds to a lack of STZs. In the experiments, this decline is observed as the failed fraction $\beta$ decreasing as $\phi$ increases, whereby $\mathrm{QP}$ events become the prevailing type (see Fig. 11).

Alternatively, one can think about the decreasing availability of nearby configurations as a manifestation the configurational entropy: systems at lower $\phi$ are thought to have a greater configurational entropy (larger number of valid states) (EDWARDS and OAKESHOTT, 1989; BRISCOE et al., 2008). This increase may also correspond to the larger variance in the local packing density at lower $\phi$ (Puckett et al., 2010), and the associated increased occurrence of larger voids would provide a means for a sheared system to explore new configurations. A description of our experimental shear zone as a system undergoing unjamming transitions also applies. As $\phi \gg \phi_{c}$, the soft modes are no longer as common, and rather than deform, our system more often finds a boundary failure mode. Recent numerical simulations by PICA CiAmarra et al. (2010) observe many of the features described here, and point to the importance of decaying tangential interactions (perhaps indicative of force-chain buckling) immediately prior to a stickslip event. In particular, precursor bursts in microscopic dynamics suggest that the system is exploring the nearby configurations, a process fundamental to the STZ model.

A broad class of systems exhibit failure events which span a broad (power-law) range of sizes, as observed for low $\phi$ in Fig. 10a. Therefore, it is valuable to compare more than just a single powerlaw exponent. Avalanche models (for a review, see BEN-Zion et al., 2010) make a set of related predictions relevant to our experiments, among them the cumulative size $(\Delta X)$ distribution with power-law exponent -2.5 , as seen for our low- $\phi$ data. A key difference between theory and our experiment, however, is that a power-law distribution of duration $\Delta T$ would also be expected, but this is not observed here. Notably, numerical simulations in DAHMEN et al. (1998, 2009a, b) exhibit periodicity-switching between AP and QP behaviors, as seen here. In both the experiment and the model, the QP behavior dominates as the system retains a larger fraction of the stress during failure. At large $\phi$, there is a faster strengthening of the system (see Fig. 10b). Since it becomes increasingly difficult to find valid states as $\phi$ approaches $\phi_{\mathrm{RCP}}$, perhaps the local failures required to form AP events become inaccessible. However, despite the many observed relationships between granular kinematics and force-chain behaviors corresponding to QP/AP transitions, it remains an open question what nucleates the transition from $\mathrm{AP}$ to $\mathrm{QP}$ or vice versa.

The theoretical frameworks described above provide promising avenues for exploring localization, periodicity-switching, and other important stick-slip dynamics. However, in order to describe the faulting process once the granular material is in motion, it will be necessary to develop a description of the rheology of the moving material. This is especially important in order to generate stress-strain-time predictions that can be tested with geophysical observations. One approach recently adopted by LAVIER and BENNETT (2010a, b) is to consider the stick-slip motion of faults in light of the viscoelastic rheology of a fluid-filled material between elastic blocks or plates. The granular gouge is taken to have an effective rheology of the type described by recent work in granular physics (JOP et al., 2006; Forterre and PouliQuen, 2008; PouliQuen and Forterre, 2009). The resulting model takes the form of a damped, driven harmonic oscillator equation (DDHO) for which damping arises from an effective viscosity $\eta$ of the flowing particles, and an effective stiffness provided by both the driving plates/blocks and the granular material within the gouge. 
In the viscosity model, during the stick phase (interval $I$ ) the system is jammed and stores energy as it is sheared. During the slip phase, the frictional dissipation within the material works to arrest the motion; simultaneously, the system is also subject to a decreasing level of stress. Thus, the event statistics $\Delta X$ and $\Delta T$ (as well as the particle-scale kinematics), provide information about the rheology of the unjammed state. In the context of our experiments, we can estimate the $\phi$-dependence of the damping coefficient via the $\phi$-dependent event duration $\langle\Delta T\rangle$. A constant driving stiffness is provided by the pulling spring, and the material stiffness is measured by $K_{\text {eff }}(\phi)$. Following Lavier and Bennett (2010a), this interpretation results in a DDHO equation of the form $\ddot{X}+D \dot{X}+\omega_{0}^{2}\left(X+X_{f}\right)=0$, with natural frequency $\omega_{0} \propto \sqrt{K_{\text {eff }}}$ (also governed by effective mass $m$ ) and damping constant $D \propto \frac{1}{\eta}$ (also with some geometric prefactors). The system is underdamped, and has a frequency of damped oscillations $\omega_{d}=$ $\sqrt{A K_{\text {eff }}(\phi)-\frac{B}{\eta(\phi)}^{2}}$, where $A, B$ are $\phi$-independent constants and both $K_{\text {eff }}$ and $\eta$ are $\phi$-dependent. The interval $I$ between events is $2 \pi / \omega_{d}$.

As observed in Fig. 10c, the stiffness $K_{\text {eff }}$ increases with increasing $\phi$ (which increases $\omega_{0}$ ) while $\langle 1 / \Delta T\rangle \propto \eta$ decreases. This latter effect may arise because at higher $\phi$ the frictional contacts are in motion largely along a single line of contacts at the imposed fault, rather than throughout the the bulk (see Fig. 11), and thus fewer contacts are involved in slowing down the system. Together, the trends in $K_{\text {eff }}(\phi)$ and $\eta(\phi)$ have opposite effects on $\omega_{d}$. This may account for the observation of only small changes in $\langle I\rangle$ over the range of parameters in these experiments.

We can further interpret the model to understand the aperiodicity in $I$. Due to the irregular kinematics and periodicity-switching, $\eta$ and $K_{\text {eff }}$ will not be the same for all events. Those states for which a nearby soft mode (see jamming model description above) is accessible/nearby will have lower $K_{\text {eff }}$ and larger $\eta$ than the average. Those states which can only find boundary failure modes will have approximately constant $K_{\text {eff }}$ and $\eta$. Systems prepared at high $\phi$ exhibit largely periodic events and largely boundary failure. In contrast, systems prepared at low $\phi$ exhibit local failure (see image in Fig. 10b) as well as boundary failure. These multiple modes of failure are consistent with the increased variability in $I$ (more AP behavior) observed for low $\phi$.

These interpretations of the experiments provide a valuable link between current granular physics and fault mechanics. The experiments also demonstrate that many of the stick-slip phenomena only recently observed in nature may originate, at least partly, via the granular mechanics of fault zones. One promising route towards a field-based hypothesis test is to consider that the relative amounts of localized (e.g. shear planes) and distributed (e.g. cataclastic foliation) fault structure might be productively interpreted in the context of boundary versus local failure modes. The two modes might be recorded in the geologic record via signatures of force-chains at the grain scale (EIchнubl et al., 2010), or manifest themselves in predictably different geodetic or seismological signatures for a given fault structure.

\section{Conclusion}

We address questions surrounding the rheology, periodicity and seismicity associated with tectonic fault slip using laboratory experiments which produce stick-slip events on a photoelastic granular aggregate. By loading the aggregate under a constant volume condition, we were able to monitor both particle-scale kinematics and force-chain dynamics for different packing densities. Key results include: (1) the effective stiffness $K_{\text {eff }}$ of the aggregate increases with $\phi$; (2) the fraction $\alpha$ of quasi-periodic events increases with $\phi$; (3) as $\phi$ increases, the aperiodic events exhibit increasingly longer intervals, while the quasi-periodic events retain the characteristic timescale; (4) these differences in periodicity manifest themselves in local kinematics; and (5) at both high and low $\phi$, the aperiodic events can involve interparticle slips between a significantly larger fraction of particles than the quasi-periodic events. The patterns of localized events have features in common with shear transformation zone theory, as well as the statistics of stick-slip events predicted by avalanche/crackling theory. We interpret the $\phi$ dependent kinematics in light of configurational restrictions on deformation as the packing density of 
the material increases. The time scale of the periodicity of the events can described by a damped, driven, harmonic oscillator where $\phi$ governs the effective viscosity and stiffness. The experiments therefore provide an important link between granular physics and fault mechanics models, especially bearing on aseismic creep vs. seismic slip, and periodic vs. aperiodic fault slip. Future tests of the hypothesis that changing force-chain dynamics with packing density lead to different effective granular viscosities and different stick-slip responses may be possible via study of natural fault structure in relation to slip dynamics.

\section{Acknowledgments}

We are grateful to Karin Dahmen and Luc Lavier for sharing key modeling results, and to Stefanos Papanikolaou and James Sethna for sharing the Wiener filtering technique. Three anonymous reviewers contributed to the revision of the manuscript. KLF and KED have been supported by a North Carolina State University FRPD and NSF CAREER award DMR0766743. LD and NWH have been supported by the University of Texas Institute for Geophysics (UTIG) under an Innovation and Opportunity grant; this is UTIG contribution \#2315.

\section{REFERENCES}

ABE S, MAIR K. Effects of gouge fragment shape on fault friction: New $3 D$ modeling results. Geophysical Research Letters 36:L23302, 2009.

Aharonov E, Sparks D. Rigidity phase transition in granular packings. Physical Review E 60:6890-6896, Dec 1999.

alonso-Marroquin F, Vardoulakis I, Herrmann HJ, Weatherley D, Mora P. Effect of rolling on dissipation in fault gouges. Physical Review E 74(3):031306, 2006.

Anthony JL, Marone C. Influence of particle characteristics on granular friction. Journal Of Geophysical Research-Solid Earth, 110(B8):B08409, Aug 192005.

BEN-Zion Y. Collective behavior of earthquakes and faults: Continuum-discrete transitions, progressive evolutionary changes, and different dynamic regimes. Rev. Geophys 46:-, December 2008.

Ben-Zion Y, Dahmen KA, Uhl JT. A unifying phase diagram for the dynamics of sheared solids and granular materials. 2010. Submitted to Pure and Applied Geophysics.

Beroza GC, Ide S (2009) Deep tremors and slow quakes. Science, 324(5930):1025-1026.
Blair D, Dufresne E. Matlab particle tracking code repository. Particle-tracking code available at http://physics.georgetown. edu/matlab/.

Bretz M, Zaretzki R, Field SB, Mitarai N, Nori F. Broad distribution of stick-slip events in slowly sheared granular media: table-top production of a Gutenberg-Richter-like distribution. Europhysics Letters 74(6):1116-1122, 2006.

Briscoe C, Song C, Wang P, Makse HA. Entropy of jammed matter. Physical Review Letters, 101(18):188001, 2008.

Brodsky EE, Mori J. Creep events slip less than ordinary earthquakes. Geophysical Research Letters 34(16):5, 2007.

Brudzinski MR, Allen RM. Segmentation in episodic tremor and slip all along Cascadia. Geology 35(10):907-910, 2007.

CARLSON JM. Two-dimensional model of a fault. Physical Review A 44(10):6226-6232, 1991.

Cates ME, Wittmer JP, Bouchaud JP, Claudin P. Jamming, force chains, and fragile matter. Physical Review Letters 81:18411844, 1998.

Chen KH, NAdeau RM, Rau RJ. Towards a universal rule on the recurrence interval scaling of repeating earthquakes?. Geophysical Research Letters 34(16):5, 2007.

Dahmen K, ERtas D, Ben-Zion Y. Gutenberg-richter and characteristic earthquake behavior in simple mean-field models of heterogeneous faults. Physical Review E 58(2):1494-1501, 1998.

Dahmen KA, Ben-Zion Y, UhL JT. Micromechanical model for deformation in solids with universal predictions for stress-strain curves and slip avalanches. Physical Review Letters 102(17): 175501, 2009a.

Dahmen KA, BEN-Zion Y, UhL JT. A simple analytic theory for the statistics of avalanches in sheared granular materials, with connections to plasticity and earthquakes. Submitted., 2009b.

DANIELS KE, HAYMAN NW. Force chains in seismogenic faults visualized with photoelastic granular shear experiments. Journal of Geophysical Research, 113:B11411, 2008. Movies available at http://www.agu.org/journals/jb/jb0811/2008JB005781/ displays.shtml.

DANTU P. Utilisation de reseaux pour l'étude experimentale des phenomenes elastiques et plastiques. Comptes Rendus Hebdomadaires Des Seances De L Academie Des Sciences 239(25): 1769-1771, 1954.

Daub EG, CARlson JM. Stick-slip instabilities and shear strain localization in amorphous materials. Physical Review E 80(6):066113, Dec 2009.

Daub EG, CARLson JM. Friction, fracture, and earthquakes. Annual Review of Condensed Matter Physics, 1, 2010.

Daub EG, Manning ML, Carlson JM. Pulse-like, crack-like, and supershear earthquake ruptures with shear strain localization. Journal Of Geophysical Research-solid Earth 115:B05311, May 182010.

Drescher A, de Josselin de Jong G. Photoelastic verification of a mechanical model for flow of a granular material. Journal Of The Mechanics And Physics Of Solids, 20:337-\&, 1972.

Edwards SF, OAKeshott RBS. Theory of powders. Physica A 157:1080-1090, 1989.

Eichnubl P, Hooker JH, Laubach SE. Pure and shear-enhanced compaction bands in Aztec Sandstone. Journal of Structural Geology 32:1873-1886, 2010.

FALK ML, LANGER JS. From simulation to theory in the physics of deformation and fracture. MRS Bulletin 25:40-45, May 2000. 
Fenistein D, van Hecke M. Kinematics - wide shear zones in granular bulk flow. Nature 425:256, Sep18 2003

Forterre Y, Pouliquen O. Flows of dense granular media. Annual Review Of Fluid Mechanics 40:1-24, 2008.

GoldFinger C, Nelson CH, Johnson JE. Holocene earthquake records from the Cascadia subduction zone and northern San Andreas Fault based on precise dating of offshore turbidites. Annual Review of Earth and Planetary Sciences 31:555-577, 2003.

Howell D, Behringer RP, Veje C. Stress fluctuations in a $2 d$ granular Couette experiment: A continuous transition. Physical Review Letters 82:5241-5244, Jun 281999.

Ide S, Beroza GC, Shelly DR, Uchide T. A scaling law for slow earthquakes. Nature 447(7140):76-79, May 32007.

Jop P, Forterre Y, Pouliquen O. A constitutive law for dense granular flows. Nature 441:727-730, 8June 2006.

Kohlstedt DL, Evans B, Mackwell SJ. Strength of the lithosphereconstraints imposed by laboratory experiments. Journal of Geophysical Research-Solid Earth 100(B9):17587-17602, 1995.

Luc L. LAVIER and Richard A. BENNETT. A model for ductile shear initiated by shear fracture: Application to slow slip events. 2010. Submitted.

Luc L. LAVIER and Richard A. BENNETT. A model for ductile shear initiated by shear fracture: Application to slow slip events. EOS Trans. AGU, Fall meeting suppl., Abstract T51F-06, 2010.

LiU AJ, NAGEL SR. The jamming transition and the marginally jammed solid. Annual Review of Condensed Matter Physics, 2010.

Liu CH, Nagel SR, Schecter DA, Coppersmith SN, Majumdar S, NaraYan O, Witten TA. Force fluctuations in bead packs. Science 269(5223):513-515, Jul 281995.

MAIR K, HAZZARD JF. Nature of stress accommodation in sheared granular material: Insights from $3 d$ numerical modeling. Earth And Planetary Science Letters 259(3-4):469-485, Jul 302007.

MARONE C. Laboratory-derived friction laws and their application to seismic faulting. Annual Review Of Earth And Planetary Sciences 26:643-696, 1998.

Miller B, O'Hern C, Behringer RP. Stress fluctuations for continuously sheared granular materials. Physical Review Letters 77:3110-3113, Oct 71996.

Miller MM, Melbourne T, Johnson DJ, Sumner WQ. Periodic slow earthquakes from the Cascadia subduction zone. Science 295(5564):2423-2423, 2002.

Mogi K. Recent earthquake prediction research in Japan. Science 233(4761):324-330, 1986.

MOORE DE, RyMer MJ. Talc-bearing serpentinite and the creeping section of the San Andreas fault. Nature 448:795-797, 2007

Morgan JK. Particle dynamics simulations of rate- and statedependent frictional sliding of granular fault gouge. Pure And Applied Geophysics 161(9-10):1877-1891, Oct 2004.

Murray J, Segall P. Testing time-predictable earthquake recurrence by direct measurement of strain accumulation and release. Nature 419(6904):287-291, 2002.

Nasuno S, Kudrolli A, Gollub JP. Friction in granular layers: Hysteresis and precursors. Physical Review Letters 79:949-952, Aug 41997.

Nasuno S, Kudrolli A, BAK A, Gollub JP. Time-resolved studies of stick-slip friction in sheared granular layers. Physical Review E 58:2161-2171, Aug 1998

O'Hern CS, Langer SA, Liu AJ, NAGel SR. Random packings of frictionless particles. Physical Review Letters 88:075507, Feb 18 2002.
O’Hern CS, Silbert LE, Liu AJ, Nagel SR. Jamming at zero temperature and zero applied stress: The epitome of disorder. Physical Review E 68:011306, Jul 2003.

ONODA GY, LinIGER EG. Random loose packings of uniform spheres and the dilatancy onset. Phys. Rev. Lett 64(22):27272730, May 1990.

Papanikolaou S, Bohn F, Sommer RL, Durin G, Zapperi S, Sethna JP. Beyond power laws: Universality in the average avalanche shape. 2010. doi:10.1038/nphys 1884 .

Peng T. Detect circles with various radii in grayscale image via Hough Transform. Particle-tracking code available at http://www.mathworks.com/matlabcentral/fileexchange/9168.

Pica Ciamarra M, Lippiello E, Godano C, de Arcangelis L. Unjamming dynamics: The micromechanics of a seismic fault model. Phys. Rev. Lett 104(23):238001, Jun 2010.

PouliQuen O, Forterre Y. A non-local rheology for dense granular flows. Philosophical Transactions of the Royal Society A 367(1909):5091-5107, 2009.

Puckett JG, Lechenault F, Daniels KE. Local origins of volume fraction fluctuations in dense granular materials. 2010. Submitted to Physical Review E: arXiv/1006.3790

Rogers G, Dragert H. Episodic tremor and slip on the Cascadia subduction zone: the chatter of silent slip. Science 300(5627): 1942-1943, 2003.

SAFFER DM, MARONE C. Comparison of smectite- and illite-rich gouge frictional properties: application to the updip limit of the seismogenic zone along subduction megathrusts. Earth and Planetary Science Letters 215(1-2):219-235, 2003.

SAMMIS CG, KING GCP. Mechanical origin of power law scaling in fault zone rock. Geophysical Research Letters 34(4):L04312, Feb 282007.

Scharer KM, Biasi GP, Weldon RJ, Fumal TE. Quasi-periodic recurrence of large earthquakes on the southern San Andreas fault. Geology 38(6):555-558, 2010.

SChleicher AM, van der Pluijm BA, Warr LN. Nanocoatings of clay and creep of the San Andreas fault at Parkfield, California. Geology 38:667-670, 2010.

$\mathrm{ScHOLz} \mathrm{CH}$. The frequency-magnitude relation of microfracturing in rock and its relation to earthquakes. Bulletin of the Seismological Society of America 58:399-415, 1968.

Scholz CH. Earthquakes and friction laws. Nature 391(6662): 37-42, 1998.

SCHWARTZ DP, COPPERSMITH KJ. Fault behvaior and characteristic earthquakes-examples from the Wasatch and San-Andreas fault zones. Journal of Geophysical Research 89(NB7):5681-5698, 1984.

ScotT GD, KiLGOUR DM. Density of random close packing of spheres. Journal Of Physics D-applied Physics 2(6):863-\&, 1969.

SEgall P, Rice JR. Dilatancy, compation, and slip instability of a fluid-infiltrated fault. Journal of Geophysical Research-Solid Earth 100(B11):22155-22171, 1995.

Segall P, Rubin AM, Bradley AM, Rice JR. Dilatant strengthening as a mechanism for slow slip events. Journal of Geophysical Research-Solid Earth. 2011. doi:10.1029/2010 JB007449.

Sethna JP, Dahmen KA, Myers CR. Crackling noise. Nature 410(6825):242-250, Mar 82001.

SILBERT LE. Jamming of frictional spheres and random loose packing. Soft Matter 6:2918-2924, 2010.

SMITH SAF, FAULKNER DR. Laboratory measurements of the frictional properties of the Zuccale low-angle normal fault, Elba 
Island, Italy. Journal of Geophysical Research-Solid Earth 115:17, 2010.

STEIN RS, KIng GCP, Lin J. Stress triggering of the 1994 M=6.7 Northridge, California, earthquake by its predecessors. Science 265(5177):1432-1435, 1994.

ToRDESILLAS A. Force chain buckling, unjamming transitions and shear banding in dense granular assemblies. Philosophical Magazine 87(32):4987-5016, 2007.

Tordesillas A, Muthuswamy M. On the modeling of confined buckling of force chains. Journal of the Mechanics and Physics of Solids 57(4):706-727, April 2009.

Torquato S, Truskett TM, Debenedetti PG. Is random close packing of spheres well defined? Physical Review Letters 84(10):2064-2067, Mar 2000. van HECKE M. Granular matter - a tale of tails. Nature 435:1041-1042, Jun 232005.

van HeCKe M. Jamming of soft particles: Geometry, mechanics, scaling and isostaticity. Journal of Physics: Condensed Matter 22:033101, 2010.

WinTsCh RP, Christoffersen R, KRONENBERG AK. Fluid-rock reaction weakening of fault zones. Journal of Geophysical Research-Solid Earth, 100(B7):13021-13032, 1995.

Wyart M. On the rigidity of amorphous solids. Annales De Physique 30(3):1, May-jun 2005.

Yu P. Stick-slip in a $2 D$ granular medium. PhD thesis, Duke University, 2008.

(Received July 7, 2010, revised November 18, 2010, accepted November 22, 2010) 